\title{
ДОСТИЖЕНИЯ И ПЕРСПЕКТИВЫ ИЗГОТОВЛЕНИЯ ЗУБНЫХ ПРОТЕЗОВ НА ИМПЛАНТАХ САD/САМ ДЛЯ ПАЦИЕНТОВ С ЧАСТИЧНОЙ АДЕНТИЕЙ (ОБЗОР ЛИТЕРАТУРЫ)
}

\section{ACHIEVEMENTS AND PROSPECTS FOR MANUFACTURE OF DENTISTS ON CAD / CAM IMPLANTS FOR PATIENTS WITH PARTIAL ADDITION (REVIEW OF LITERATURE)}

\author{
А.В. Ефимин ${ }^{1,2}$, К.Н. Барковец ${ }^{1,2}$ \\ A.V. Efimin ${ }^{1,2}$, K.N. Barkovec ${ }^{1,2}$ \\ ${ }^{1} \mathrm{OOO}$ «Дента Люкс», \\ Россия, 433870, Ульяновская область, р. п. Новоспасское, ул. 40 лет Победы, д. 45 \\ ${ }^{2}$ Белгородский государственный национальный исследовательский университет, \\ Россия, 308015, г. Белгород, ул. Победы, д. 85 \\ ${ }^{1}$ DENTA Lux LLC, \\ 4540 let Pobedy St., Novospasskoye work settlement, Ulyanovsk region, 433870, Russia \\ ${ }^{2}$ Belgorod National Research University, \\ 85 Pobedy St., Belgorod, 308015, Russia \\ E-mail: a-efimin@yandex.ru
}

\begin{abstract}
Аннотация
Развитие медицинской науки в целом, а в частности - стоматологии, на сегодняшний день характеризуется процессом взаимоинтеграции с техническими инновациями с целью повышения точности, эффективности лечебно-диагностического процесса, а также оптимизации работы системы здравоохранения. Статья содержит обзор публикаций по проблеме современного состояния и перспектив изготовления зубных протезов на имплантах $\mathrm{CAD} / \mathrm{CAM}$ для пациентов с частичной адентией. В процессе аналитического обзора установлено, что на сегодняшний день активно применяется в различных сферах экономической деятельности система автоматизированного проектирования (САПР). Многие протезы изготавливаются с помощью систем CAD/CAM с использованием различных типов материалов, таких как фарфор, композитная смола и металлические блоки. Даже такие материалы, как диоксид циркония, которые не могли быть изготовлены обычными способами ранее из-за технических ограничений, теперь могут быть изготовлены этими системами. Кроме того, постоянно идет разработка новых материалов для усовершенствования рабочей платформы за счет сочетания CAD/CAM-технологий, высокопрочной керамики и современных композитных цементов, плавиковой кислоты, сандбластинга. Имеющиеся данные обеспечивают многообещающие результаты для реставраций на основе имплантатов, изготовленных с использованием CAD/CAM.
\end{abstract}

\footnotetext{
Abstract

The development of medical science in general, and in particular dentistry, today is characterized by a process of mutual integration with technical innovations in order to improve the accuracy, efficiency of the treatment and diagnostic process, as well as optimizing the functioning of the healthcare system. The article contains a review of publications on the current state and prospects of manufacturing dentures on $\mathrm{CAD} / \mathrm{CAM}$ implants for patients with partial adentia. In the process of the analytical review it was established that today the computer-aided design (CAD) system is actively used in various fields of economic activity. Many prostheses are made using CAD/CAM systems using various types of materials such as porcelain, composite resin and metal blocks. Even materials such as zirconia, which could not be manufactured by conventional methods previously due to technical limitations, can now be made by these systems. In addition, new materials are constantly being developed to improve the working platform
} 
through a combination of CAD/CAM technology, high-strength ceramics and modern composite cements, hydrofluoric acid, sand blasting. The available data provide promising results for CAD/CAMbased implant restorations.

Ключевые слова: частичная адентия, зубное протезирование, CAD/CAM, система автоматизированного проектирования, система автоматизации производства.

Keywords: partial adentia, dental prosthetics, CAD/CAM, computer-aided design system, production automation system.

\section{Введение}

Растущий спрос на автоматизацию является одним из основных факторов, стимулирующих рост рынка программной инженерии. Такие приложения, как автоматизированное проектирование (CAD) и автоматизированное производство (CAM), становятся все более популярными, используются для проектирования изделий и программных производственных процессов, таких как обработка с ЧПУ (компьютерное числовое управление). За последние пару десятилетий было внедрено много передовых компьютерных и лабораторных систем CAD/CAM - систем автоматизации производства [De Franca et al., 2019; Negm et al., 2019]. Интегрированные CAD/CAM-системы - это максимально наукоемкие продукты, постоянно развиваются и включают в себя новые знания в области моделирования и обработки материалов. В программном обеспечении САМ используются модели и сборки для создания траекторий инструментов для приводных машин, которые превращают конструкции в физические детали [Fasbinder et al., 2016; Vech et al., 2017]. Программное обеспечение CAD обычно используется для твердотельного моделирования, моделирования поверхностей, сборки, черчения, детализации и обратного проектирования. Приложения САМ обычно включают плоттеры, лазерную резку, 25D-фрезерование, 3D-фрезерование, токарную обработку и 3D-печать [Матко, 2015]. Основные преимущества использования программного обеспечения для проектирования, такие как CAD/CAM, включают улучшенную точность, расширенный диапазон дизайнерских идей, простоту модификации, повторяемость вывода, качество вывода и снижение потерь [Batson et al., 2014; Joda et al., 2016]. Комбинация CAD и CAM обеспечивает автоматический переход от проектирования к производству программ CAD/CAM, также все чаще и чаще используются в стоматологической визуализации [Davidowitz et al., 2011; Carneiro et al., 2020].

Распространенность частичной и полной адентии среди взрослых является одной из ключевых проблем современной медицины. В связи с этим ВОЗ определила прерогативу развития стоматологического здоровья для обеспечения сохранения как минимум 20 естественных зубов у лиц старше 80 лет с помощью комплексных программ профилактики и профилактических мероприятий на разных уровнях системы стоматологической помощи [Papadiochou et al., 2018]. Однако, несмотря на ключевую роль профилактики, лечение пациентов с уже имеющимися частичными дефектами зубных рядов остается одной из самых распространенных манипуляций в ортопедической практике. В современной имплантологии широкий интерес получили передовые технологии визуализации и CAD/CAM.

Цель исследования - анализ литературных источников с детальным изучением современного состояния и перспектив изготовления зубных протезов на имплантах $\mathrm{CAD} / \mathrm{CAM}$ для пациентов с частичной адентией.

\section{Материалы и методы исследования}

Проведен обзор и анализ научно-медицинской литературы за период с 2015 по 2020 гг. из баз данных Scopus, The Cochrane Library, Global Health, CyberLeninka, РИНЦ. Сосредоточено внимание на современном состоянии и перспективах изготовления зубных протезов на имплантах $\mathrm{CAD} / \mathrm{CAM}$ для пациентов с частичной адентией. 


\section{Результаты исследования и их обсуждение}

Система CAD представляет собой технологию, построенную на так называемой системе геометрического моделирования, которая работает с трехмерными объектами и прежде всего обеспечивает фазу получения исходных данных из тканей протезного поля [Наумович и др., 2016]. Дальше система CAD работает в аналитической фазе проектирования, которая заключается в анализе параметров будущего протеза и изготовлении аналитической модели будущей конструкции с помощью средств автоматизированного конструирования (САЕ). Когда аналитическая модель будущей конструкции сформирована, начинается фаза оценки проекта и прототипирования, то есть конструируется виртуальный прототип («цифровая копия») конструкции [Algazali., 2016; Al-Merikhi et al., 2018]. В настоящее время сконструирован прототип с помощью программных пакетов, который обрабатывает и генерирует коды для автоматизированной подготовки производства (CAM). Важнейшая функция системы CAD заключается в определении геометрии конструкций, которая, в свою очередь, определяет дальнейшую работу систем проектирования (САE) и изготовления (САM) готовой конструкции [Cathedial et al., 2013].

Система автоматизированного конструирования (САЕ) - это технология, которая использует компьютерные системы для анализа геометрии $\mathrm{CAD}$, моделирования и изучения будущей конструкции, усовершенствования и оптимизации конструктивных особенностей (т. н. «виртуальная шлифовка», или функция предварительного просмотра результатов) трехмерной цифровой модели, когда с помощью компьютерной мыши или трекбола определяются и убираются мелкие погрешности сканирования или моделирования (дизайна) конструкции [Lebon et al., 2016].

Система CAD получает данные с помощью сканера протезного поля, или дигитайзера. Сканеры различают по типам методик получения графической информации - бесконтактные, к которым относят электронно-оптические и лазерные системы, и контактные, в которых применяется механический контактный элемент, который сканирует поверхность опорного зуба и прилегающих тканей. С помощью таких сканеров получается трехмерный виртуальный отпечаток [Saponaro et al., 2016]. Сканирующие системы могут применяться как в полости рта, так и на моделях челюстей. Внутриротовые оптические сканеры получили меньшее распространение, обусловленное возможным негативным влиянием «человеческого» фактора (невозможность статической фиксации сканера в полости рта вследствие дрожания рук; избыточное количество, недостаток или повышенная влажность антибликового порошка на участке, подлежащем сканированию; отражение света от металлических деталей и т. п.) [Petersen et al., 2005].

Компьютеры используются для сбора данных, проектирования и производства широкого спектра продуктов в системах CAD/CAM [Разуменко, 2016]. Эти системы долгое время использовались в промышленности, но они не были доступны для применения в стоматологии до 1980-х годов [Карапетян и др., 2018], хотя история развития систем $\mathrm{CAD} / \mathrm{CAM}$ уходит своими корнями в древние времена. Еще Леонардо да Винчи указал в своих работах на использование современной графической конвенции. Эвклида Александрийского, великого математика, действительно можно считать человеком, стоящим за современным программным обеспечением САПР, потому что современное программное обеспечение САПР основано именно на аксиомах и постулатах этого ученого [Иванникова и др., 2018].

Первый САМ с цифровым управлением, который получил название «Pronto», разработал Патрик Дж. Ханратти, которого называют отцом CAD/CAM. В течение последних 150 лет методы ручного производства постепенно замещались механизированными методами, которые с каждым годом становятся все сложнее, станки разного назначения объединяются, создаются автоматизированные линии [Акулин и др., 2019].

Внедрение CAD/CAM в стоматологии начинается с работы Франсуа Дюре, который разработал ее концепцию в 1970 году. Впоследствии разработкой технологии 
$\mathrm{CAD} / \mathrm{CAM}$ для стоматологии занимались разные исследовательские группы [Алиева и др., 2019]. За последние десятилетия новые разработки привели к успеху современных стоматологических CAD/CAM-технологий. Управляемая имплантация с использованием этих передовых технологий имеет решающее значение, в том числе в реабилитации пациентов с частичной адентией [Arberg et al., 2016].

Так, например, S. Papadiochou et al. проведена оценка отличий предельной адаптации $\mathrm{CAD} / \mathrm{CAM}$-одиночных коронок, фиксированных зубных протезов и фиксированных зубных протезов с имплантатами или их инфраструктуры от той, которая была получена другими методами изготовления с использованием аналогичного восстановительного материала, и наличие ее зависимости от типа восстановительного материала. Большинство реставраций/инфраструктур CAD/CAM, по данным автора, находились в пределах клинически приемлемого диапазона предельного расхождения (MD). На производительность системы CAD/CAM относительно предельной адаптации влияет восстановительный материал. По сравнению с CAD/CAM, большинство коронок из дисиликата лития, подвергнутых термическому прессованию, имеют равные или меньшие значения MD. Коронки со скользящим литьем демонстрировали ту же или лучшую предельную точность, чем изготовленные с использованием CAD/CAM. Инфраструктуры кобальт-хромовых и титановых имплантатов, изготовленные с использованием CAD/CAM-системы, выявила меньшие значения $\mathrm{MD}$, чем диоксид циркония. Большинство кобальт-хромовых реставраций/инфраструктур, изготовленных с помощью DMLS, показали лучшую предельную точность, чем те, которые были изготовлены методом литья. По сравнению с фрезерным копированием, большинство реставраций/инфраструктур из диоксида циркония, полученных с помощью фрезерования $\mathrm{CAD} / \mathrm{CAM}$, показали лучшую маргинальную адаптацию. При этом невозможно сделать четкие выводы о превосходстве фрезерования CAD/CAM над техникой литья и DMLS в отношении предельной адаптации [Papadiochou et al., 2018].

Сравнение адаптации основания зубного протеза между CAD/CAM и традиционными методами изготовления, проведенное B.J. Goodacre et al., показало, что процесс изготовления CAD/CAM был наиболее точным и воспроизводимым способом изготовления зубных протезов по сравнению с технологиями обработки основы и прессования, заливки и литья под давлением [Goodacre et al., 2016].

P. Ahlholm et al., в свою очередь, отмечено, что методы цифрового оттиска являются клинически приемлемой альтернативой традиционным методам оттиска при изготовлении коронок и коротких фиксированных зубных протезов (FDP). Для изготовления коронок с имплантатами и систем FDP цифровые оттискные системы также обеспечивают клинически приемлемую посадку. Методы цифрового оттиска быстрее и могут сократить время операции [Ahlholm et al., 2018].

Анализ полученных результатов показывает, что преимуществом CAD/CAMсистем над традиционными технологиями изготовления зубных протезов является возможность получения конструкций протезов более высокого качества за менее короткое время и с меньшими трудозатратами [Алиева, 2019; Azarbal, 2018]. Следует отметить, что принципиальным отличием CAD/CAM-систем от традиционных технологий изготовления зубных протезов является практически полное нивелирование влияния «человеческого» фактора точности изготовления конструкции, поскольку в каждом клиническом случае виртуальное моделирование (CAD) будущей конструкции осуществляется на модели зуба, полученной методиками контактного или оптического сканирования в трехмерном пространстве с последующим изготовлением протеза с помощью САМ, которая состоит из аппаратной системы производства и органов управления [Горелова и др., 2016].

$\mathrm{CAD} / \mathrm{CAM}$ позволила стоматологам использовать возможности компьютера для разработки и проектирования деталей для реставрации зубов. Появление современного дизайна имплантата и усовершенствованных технологий обработки поверхности позволило разработать новые методы реставрации, которые сокращают общее время лечения пациента [Апресян, 2019; Homaei et al., 2016]. Новейшие технологии сканирования и 
CAD/CAM-производства, позволили изготовить индивидуальную реставрацию зубов с высокой точностью и безошибочной посадкой.. За последние годы на рынке выросли приложения CAD/CAM. В настоящее время существует множество коммерческих источников, которые могут производить чисто CAD/CAM-стержни и каркасы, или фрезерованные CAM-структуры для протезов имплантатов [Vech et al., 2017].

Крупными игроками на мировом рынке стоматологических CAD/CAM являются Straumann (Великобритания), PLANMECA OY (Хельсинки, Финляндия), Danaher Corporation (СШA), Dentsply Sirona (СШA), Align Technology, Inc. (СШA), Roland DG Corporation (Япония) и DATRON AG (Великобритания) [Infante et al., 2014]. Североамериканский регион лидирует на рынке стоматологических CAD/CAM в 2019 году, за ним следуют Европа, АРАC, MЕА и Южная Америка [Faeghinejad et al., 2019]. Ожидается, что мировой рынок стоматологических CAD/CAM достигнет к 2027 году 33,00 млн долл. Рост рынка будет способствовать увеличению доли стоматологических реставраций на основе CAD/CAM, росту спроса на современные стоматологические технологии. Ожидается, что Европа будет занимать наибольшую долю на мировом рынке стоматологических $\mathrm{CAD} / \mathrm{CAM}$ в течение прогнозируемого периода и будет занимать $42,10 \%$ рынка. Это может быть связано с растущей осведомленностью о стоматологических CAD/CAM, растущей поддержкой со стороны правительства и наличием большой гериатрической популяции [Ханахмедов, 2018]. Однако высокая стоимость стоматологических систем CAD/CAM, как ожидается, одновременно будет препятствовать росту рынка в течение прогнозируемого периода [Käsemodel et al., 2020].

Тем не менее новые технологии будут продолжать расширять границы использования CAD/CAM-систем. Акцент ставится на внутриротовом сборе данных. Это может привести в конечном итоге к устранению изготовления слепков и моделей [Сунарчин, 2018]. Вероятно, будущие дигитайзеры или сканеры будут более надежными, обеспечат точный сбор данных, несмотря на индивидуальные различия клиентов. Это означает, что данные могут быть отправлены непосредственно CAD/CAM-системой без интерпретации техником или врачом [Boutros, 2017]. Программное обеспечение САПР является относительно разработанным и, скорее всего, кардинально не изменится. Тем не менее вероятные улучшения могут включать в себя более простой пользовательский интерфейс и интеграцию виртуальных артикуляторов, которые облегчили бы автоматическое проектирование окклюзионной поверхности.

\section{Выводы}

Технология CAD/CAM используется для проектирования и производства различных типов изделий, включая зубное протезирование. Она используется в цифровой стоматологии для изготовления зубных протезов, таких как коронки, виниры, вкладки и накладки, фиксированные мосты, реставрации зубных имплантатов, зубные протезы и даже полной реконструкции. Использование CAD/CAM в стоматологии постоянно растет, поскольку оно обеспечивает достаточную прочность реставраций, поддерживает естественный внешний вид и повышает точность и скорость создания реставраций. Достижения стоматологической науки, появление новейших технологий позволяют на новом уровне подойти к проблеме реабилитации пациентов с частичным отсутствием зубов при помощи съемных и несъемных протезов, опирающихся на дентальные имплантаты, изготовленные с помощью системы CAD/CAM, которые помогают стоматологам предоставлять высококачественные стоматологические услуги своим пациентам. Ожидается, что сегмент стоматологических систем $\mathrm{CAD} / \mathrm{CAM}$ сохранит свое доминирующее положение благодаря расширению его применения, особенно в развитых регионах. Тем не менее следует указать на тот факт, что современные данные ограничены из-за качества доступных исследований и недостаточного количества данных о долгосрочных клинических исходах от 5 лет и более. 


\section{Список литературы}

1. Акулин А.В., Бабаева А.А. 2019. Компьютерные технологии в стоматологии. В сборнике: РОСТ - Развитие, Образование, Стратегии, Технологии. Сборник материалов II Всероссийской научно-практической конференции. С. 3-9.

2. Алиева С.П. 2019. Компьютерное моделирование при имплантации: анализ отечественного и зарубежного опыта. International Journal of Medicine and Psychology. 2 (4): 83-90.

3. Апресян С.В. 2019. Цифровое планирование ортопедического стоматологического лечения. Российский стоматологический журнал. 23 (3-4): 158-164.

4. Горелова В.А., Орехов С.Н., Матвеев С.В. 2016. СAD/CAM - технология в ортопедической стоматологии. Международный студенческий научный вестник. 4-3: 246-248.

5. Иванникова А.С., Мешков В.А., Уржумов Р.Л. 2018. Современная технология $\mathrm{CAD} / \mathrm{CAM}$ в стоматологии. В книге: Научно-исследовательская работа студентов стоматологического факультета. Материалы II научно-практической конференции студентов стоматологического факультета в рамках дисциплины «Практика. НИР». Читинская государственная медицинская академия. С. 21-23.

6. Карапетян Т.А., Перунов А.Ю. 2018. Технология CAD/CAM - ортопедическая стоматология будущего. Бюллетень медицинских интернет-конференций. 8 (2): 63-64.

7. Матко С. 2015. Определение наиболее оптимального метода заключительного этапа ортопедического лечения пациентов с помощью реставраций, изготовленных по CAD/CAMтехнологии системой Chairside. Стоматология. 6: 64-63.

8. Наумович С.С., Разоренов А.Н. 2016. CAD/CAМ-системы в стоматологии: современное состояние и перспективы развития. Современная стоматология. 4 (65): 2-9.

9. Разуменко Г.П. 2016. Значение и место CAD/CAM-технологий для отечественной ортопедической стоматологии. Актуальные проблемы гуманитарных и естественных наук. 8-2: 169-173.

10. Сунарчин Э.И., Самукова Е.А., Бабаева А.А. 2018. Использование 3D-технологий и $\mathrm{CAD} / \mathrm{CAM}$ в стоматологии. Современные научные исследования и разработки. 3 (20): 845-847.

11. Ханахмедов В.А. 2018. Уникальная технология CAD/CAM-систем в ортопедической стоматологии для изготовления каркасов несъёмных зубных протезов, анализ различных CAD/CAM-систем. Бюллетень медицинских интернет-конференций. 8 (2): 46-47.

12. Ahlholm P., Sipilä K., Vallittu P., Jakonen M., Kotiranta U. 2018. Digital and conventional impressions in fixed prosthetics: anoverview. J. Prosthodont. January; 27 (1): 35-41. DOI: 10.1111 / jopr.12527.

13. Algazali T.F. 2016. Advances in CAD/CAM technology: options for practical implementation. Journal of Prostodontic research. 60 (2): 72-84.

14. Al-Merikhi X., Yilmaz B., McGlumphy E., Brantley, Washington; Johnston W.M. 2018. Distortion of dental prosthesis frames made of titanium and zirconium dioxide made from CAD/CAM implants. J. Prosthesis. Dent., 119: 116-123.

15. Arberg D., Lauer H.K., Arberg M., Weigl P. 2016. Evaluating the suitability and effectiveness of fully ceramic restorations from CAD/CAM based on direct and indirect digitization: a doubleblind randomized clinical trial. Clin. Oral. Investig. 20 (2): 291-300.

16. Azarbal A., Azarbal M., Engelmayer R.L., Kunkel T.S. 2018. Comparison of the maximum fit of CAD/CAM crowns milled from two different materials. Journal of prosthetics. 27 (5): 421-428.

17. Batson E.R., Cooper L.F., Ducum I., Mendonca. 2014. Clinical results of three different crown systems with CAD/CAM technology. J. Prosthet. Dent. 112: 770-777.

18. Boutros S. 2017. CAD/CAM technologies help to create ideal orthopedic structures with support for implants. Dental prosthetics. 1: 19-23.

19. Carneiro P., Bezerra de Medeiros, de Souza Santos, Oliveira de Almeida. Precision of $\mathrm{CAD} / \mathrm{CAM}$ systems for manufacturing removable partial denture frames: a systematic review. J. Prosthetic. Dent. 2020, March 5. pii: S0022-3913 (20) 30018-4. DOI: 10.1016 / j. prosdent.2020.01.003.

20. Cathedial M.T., Goodacre C.J., Baba N.Z. 2013. CAD/CAM complete dentures: a review of two commercial systems of production. J. Calif. Dent. Assoc.; 41: 407-16.

21. Davidowitz G., Kotick P.G. 2011. The use of CAD/CAM in dentistry. Dental. Clinic. N. Am. 55: 559-70.

22. De Franca D.G., Morais M.H., Das Neves F.D., Barbosa G.A. 2015. The impact of $\mathrm{CAD} / \mathrm{CAM}$ on the accuracy of fitting implants made of zirconium and cobalt-chrome fixed dentures. J. Prosthet. Dent. 113: 22-28. 
23. Faeghinejad M., Proussaefs P., Al Helal A., Lozada J. 2019. Composite prosthesis CAD/CAM: a digital process for manufacturing a cement-held prosthesis made of zirconium dioxide on top of milled titanium rods with screw fixation. Int. J. Periodontics Restorative Dent. 39: 39-47.

24. Fasbinder D.J., Neiva G.F. 2016. Surface evaluation of polishing techniques for new resilient CAD/CAM restorative materials. Journal of Esthetic and Restorative Dentistry. 28(1): 56-66.

25. Goodacre B.J., Goodacre C.J., Baba N.Z., Kattadiyil M.T. 2016. Comparison of adaptation of the denture base between CAD/CAM and traditional manufacturing methods. J. Denture dent. 116 (2): 249-256. DOI: 10.1016 / j. prosdent.2016.02.017.

26. Homaei E., Farhangdoost K., Tsoi J.K.H., Matinlinna J.P., Pow E.H.N. 2016. Static and fatigue mechanical behavior of three dental CAD/CAM ceramics. J. Mech. Behav. Biomed. Mater. 7: 304-313.

27. Infante L., Yilmaz B., McGlumphy E. 2014. Manufacturing of complete removable prostheses with CAD/CAM technology. J. Prosthet. Dentistry; 111: 351-5.

28. Joda T., Ferrari M., Braegger U. 2016. Digital approach for one-step formation of the supraimplant appearance profile with an individualized CAD/CAM healing abutment. J. Prosthodont. Residence; 60: 220-223.

29. Käsemodel R.B., de Souza A.F., Voigt R. 2020. The CAD/CAM interface algorithm reduces cutting force, roughness, and processing time when milling in free form. Int. J. Adv. Manuf. Technol. https://doi.org/10.1007/s00170-020-05143-X.

30. Lebon N., Tapie L., Duret F., Attal J.P. 2016. Understanding Dental CAD/CAM for Restorations-Dental Milling Machines from a Mechanical Engineering Viewpoint. Part B: Labside Milling Machines. International Journal of Computerized Dentistry. 19(2): 115-134.

31. Negm E.E., Aboutaleb F.A., Alam-Eldein A.M. 2019. Virtual assessment of fit and accuracy in removable frames of a partial upper jaw prosthesis made of poly (etherephyrketone), made using direct and indirect CAD/CAM techniques. J. Prosthodont. 28 (7): 804-810. doi: 10.1111 / jopr.13075. Epub 2019 June 11.

32. Papadiochou S., Pissiotis A.L. 2018. Marginal adaptation and CAD/CAM technology: a systematic review of restorative materials and manufacturing technologies. Prosthetic dent. 119 (4): 545-551. doi: 10.1016/j. prosdent.2017.07.001.

33. Petersen P.E., Yamamoto T. 2005. Improving the oral health of older people: the approach of the who Global oral hygiene program. Community Dent. Oral. Epidemiol. 33: 81-92.

34. Saponaro P.S., Yilmaz B., Heshmati R.H., Mcglumfi E.A. 2016. Clinical characteristics of complete dentures made from CAD/CAM: a cross-sectional study. J. Prosthetic. Dent. 116: 431-435.

35. Vech B., GOOS-Kovacs G., Borbély J., Herman P. 2017. Comparing the accuracy of direct and indirect three-dimensional digitization processes for CAD/CAM systems-an in vitro study. Journal of orthopedic research. 61 (2): 177-184.

\section{References}

1. Akulin A.V., Babaeva A.A. 2019. Komp'yuternye tekhnologii v stomatologii [Computer technology in dentistry]. V sbornike: ROST - Razvitie, Obrazovanie, Strategii, Tekhnologii. Sbornik materialov II Vserossiiskoi nauchno-prakticheskoi konferentsii. S. 3-9.

2. Alieva S.P. 2019. Komp'yuternoe modelirovanie pri implantatsii: analiz otechestvennogo i zarubezhnogo opyta [Computer modeling during implantation: analysis of domestic and foreign experience]. International Journal of Medicine and Psychology. 2. (4): 83-90.

3. Apresyan S.V. 2019. Tsifrovoe planirovanie ortopedicheskogo stomatologicheskogo lecheniya [Digital plan orthopedic dental treatment]. Rossiiskii stomatologicheskii zhurnal. 23 (3-4): 158164.

4. Gorelova V.A., Orekhov S.N., Matveev S.V. 2016. CAD/CAM - tekhnologiya v ortopedicheskoi stomatologii [CAD/CAM technology in prosthetic dentistry]. Mezhdunarodnyi studencheskii nauchnyi vestnik. 4-3: 246-248.

5. Ivannikova A.S., Meshkov V.A., Urzhumov R.L. 2018. Sovremennaya tekhnologiya CAD/CAM v stomatologii [Urzhumov Modern CAD/CAM technology in dentistry]. V knige: Nauchnoissledovatel'skaya rabota studentov stomatologicheskogo fakul'teta. Materialy II nauchno-prakticheskoi konferentsii studentov stomatologicheskogo fakul'teta v ramkakh distsipliny «Praktika. NIR». Chitinskaya gosudarstvennaya meditsinskaya akademiya. S. 21-23. 
6. Karapetyan T.A., Perunov A.Yu. 2018. Tekhnologiya CAD/CAM - ortopedicheskaya stomatologiya budushchego [Technology of CAD/CAM prosthetic dentistry of the future]. Byulleten' meditsinskikh internet-konferentsii. 8 (2): 63-64.

7. Matko S. 2015. Opredelenie naibolee optimal'nogo metoda zaklyuchitel'nogo etapa ortopedicheskogo lecheniya patsientov s pomoshch'yu restavratsii izgotovlennykh po CAD/CAM tekhnologii sistemoi Chairside [Determination of the optimal method of the final stage of orthopedic treatment of patients with restorations made by CAD/CAM technology the Chairside system]. Stomatologiya. 6: 64-63.

8. Naumovich S.S., Razorenov A.N. 2016. SAD/CAM sistemy v stomatologii: sovremennoe sostoyanie i perspektivy razvitiya [CAD/CAM systems in dentistry: current state and prospects of development]. Sovremennaya stomatologiya. 4 (65): 2-9.

9. Razumenko G.P. 2016. Znachenie i mesto CAD/CAM tekhnologii dlya otechestvennoi ortopedicheskoi stomatologii [the Importance and place CAD/CAM technologies for domestic prosthetic dentistry]. Aktual'nye problem gumanitarnykh i estestvennykh nauk. 8 (2): 169-173.

10. Sunarchin E.I., Samukova E.A., Babaeva A.A. 2018. Ispol'zovanie 3D-tekhnologii i $\mathrm{CAD} / \mathrm{CAM} \mathrm{v}$ stomatologii [The Use of 3D technology and CAD/CAM in dentistry]. Sovremennye nauchnye issledovaniya i razrabotki. 3 (20): 845-847.

11. Khanakhmedov V.A. 2018. Unikal'naya tekhnologiya CAD/CAM sistem v ortopedicheskoi stomatologii dlya izgotovleniya karkasov nes"emnykh zubnykh protezov. Analiz razlichnykh CAD/CAM system [the Unique technology of CAD/CAM systems in prosthetic dentistry for the fabrication of frameworks of fixed dental prostheses, the analysis of different CAD/CAM systems]. Byulleten' meditsinskikh internet-konferentsii. 8 (2): 46-47.

12. Ahlholm P., Sipilä K., Vallittu P., Jakonen M., Kotiranta U. 2018. Digital and conventional impressions in fixed prosthetics: anoverview. J. Prosthodont. January; 27 (1): 35-41. DOI: 10.1111 / jopr.12527.

13. Algazali T.F. 2016. Advances in CAD/CAM technology: options for practical implementation. Journal of Prostodontic research. 60 (2): 72-84.

14. Al-Merikhi X., Yilmaz B., McGlumphy E., Brantley, Washington; Johnston W.M. 2018. Distortion of dental prosthesis frames made of titanium and zirconium dioxide made from CAD/CAM implants. J. Prosthesis. Dent., 119: 116-123.

15. Arberg D., Lauer H.K., Arberg M., Weigl P. 2016. Evaluating the suitability and effectiveness of fully ceramic restorations from CAD/CAM based on direct and indirect digitization: a doubleblind randomized clinical trial. Clin. Oral. Investig. 20 (2): 291-300.

16. Azarbal A., Azarbal M., Engelmayer R.L., Kunkel T.S. 2018. Comparison of the maximum fit of CAD/CAM crowns milled from two different materials. Journal of prosthetics. 27 (5): 421-428.

17. Batson E.R., Cooper L.F., Ducum I., Mendonca. 2014. Clinical results of three different crown systems with CAD/CAM technology. J. Prosthet. Dent. 112: 770-777.

18. Boutros S. 2017. CAD/CAM technologies help to create ideal orthopedic structures with support for implants. Dental prosthetics. 1: 19-23.

19. Carneiro P., Bezerra de Medeiros, de Souza Santos, Oliveira de Almeida. Precision of CAD/CAM systems for manufacturing removable partial denture frames: a systematic review. J. Prosthetic. Dent. 2020, March 5. pii: S0022-3913 (20) 30018-4. DOI: 10.1016 / j. prosdent.2020.01.003.

20. Cathedial M.T., Goodacre C.J., Baba N.Z. 2013. CAD/CAM complete dentures: a review of two commercial systems of production. J. Calif. Dent. Assoc.; 41: 407-16.

21. Davidowitz G., Kotick P.G. 2011. The use of CAD/CAM in dentistry. Dental. Clinic. N. Am. 55: 559-70.

22. De Franca D.G., Morais M.H., Das Neves F.D., Barbosa G.A. 2015. The impact of $\mathrm{CAD} / \mathrm{CAM}$ on the accuracy of fitting implants made of zirconium and cobalt-chrome fixed dentures. J. Prosthet. Dent. 113: 22-28.

23. Faeghinejad M., Proussaefs P., Al Helal A., Lozada J. 2019. Composite prosthesis CAD/CAM: a digital process for manufacturing a cement-held prosthesis made of zirconium dioxide on top of milled titanium rods with screw fixation. Int. J. Periodontics Restorative Dent. 39: 39-47.

24. Fasbinder D.J., Neiva G.F. 2016. Surface evaluation of polishing techniques for new resilient CAD/CAM restorative materials. Journal of Esthetic and Restorative Dentistry. 28(1): 56-66. 
25. Goodacre B.J., Goodacre C.J., Baba N.Z., Kattadiyil M.T. 2016. Comparison of adaptation of the denture base between CAD/CAM and traditional manufacturing methods. J. Denture dent. 116 (2): 249-256. DOI: $10.1016 / \mathrm{j}$. prosdent.2016.02.017.

26. Homaei E., Farhangdoost K., Tsoi J.K.H., Matinlinna J.P., Pow E.H.N. 2016. Static and fatigue mechanical behavior of three dental CAD/CAM ceramics. J. Mech. Behav. Biomed. Mater. 7: 304-313.

27. Infante L., Yilmaz B., McGlumphy E. 2014. Manufacturing of complete removable prostheses with CAD/CAM technology. J. Prosthet. Dentistry; 111: 351-5.

28. Joda T., Ferrari M., Braegger U. 2016. Digital approach for one-step formation of the supraimplant appearance profile with an individualized CAD/CAM healing abutment. J. Prosthodont. Residence; 60: 220-223.

29. Käsemodel R.B., de Souza A.F., Voigt R. 2020. The CAD/CAM interface algorithm reduces cutting force, roughness, and processing time when milling in free form. Int. J. Adv. Manuf. Technol. https://doi.org/10.1007/s00170-020-05143-x.

30. Lebon N., Tapie L., Duret F., Attal J.P. 2016. Understanding Dental CAD/CAM for Restorations-Dental Milling Machines from a Mechanical Engineering Viewpoint. Part B: Labside Milling Machines. International Journal of Computerized Dentistry. 19(2): 115-134.

31. Negm E.E., Aboutaleb F.A., Alam-Eldein A.M. 2019. Virtual assessment of fit and accuracy in removable frames of a partial upper jaw prosthesis made of poly (etherephyrketone), made using direct and indirect CAD/CAM techniques. J. Prosthodont. 28 (7): 804-810. doi: 10.1111 / jopr.13075. Epub 2019 June 11.

32. Papadiochou S., Pissiotis A.L. 2018. Marginal adaptation and CAD/CAM technology: a systematic review of restorative materials and manufacturing technologies. Prosthetic dent. 119 (4):

33. 545-551. doi: 10.1016/j. prosdent.2017.07.001.

34. Petersen P.E., Yamamoto T. 2005. Improving the oral health of older people: the approach of the who Global oral hygiene program. Community Dent. Oral. Epidemiol. 33: 81-92.

35. Saponaro P.S., Yilmaz B., Heshmati R.H., Mcglumfi E.A. 2016. Clinical characteristics of complete dentures made from CAD/CAM: a cross-sectional study. J. Prosthetic. Dent. 116: 431-435.

36. Vech B., GOOS-Kovacs G., Borbély J., Herman P. 2017. Comparing the accuracy of direct and indirect three-dimensional digitization processes for CAD/CAM systems-an in vitro study. Journal of orthopedic research. 61 (2): 177-184.

\section{Ссылка для цитирования статьи Link for article citation}

Ефимин А.В., Барковец К.Н. 2020. Достижения и перспективы изготовления зубных протезов на имплантах CAD/CAM для пациентов с частичной адентией (обзор литературы). Актуальные проблемы медицины, 43 (2): 304-312. DOI 10.18413/2687-0940-2020-43-2-304-312.

Efimin A.V., Barkovec K.N. 2020. Achievements and prospects for manufacture of dentists on CAD/CAM implants for patients with partial addition (review of literature). Challenges in Modern Medicine, 43 (2): 304-312 (in Russian). DOI 10.18413/2687-0940-2020-43-2-304-312. 